\title{
Original
}

\section{Clips metálicos de titanio: una técnica de sutura más rápida y fácil de utilizar que las suturas convencionales en autotrasplantes renales heterotópicos en cerdos en crecimiento}

\author{
$\mathrm{M}^{\mathrm{a}}$ Carmen Calles Vázquez*, Elena Abellan Rubio, Verónica Crisóstomo Ayala, \\ $\mathrm{M}^{\mathrm{a}}$ Fernanda Martín Cancho, Francisco M. Sánchez Margallo*, Jesús Usón Gargallo \\ Unidad de Microcirugía. Centro de Cirugía de Mínima Invasión “Jesús Usón”. Cáceres.
}

\begin{abstract}
Resumen
Introducción: En reconstrucciones de elementos vasculares en crecimiento y concretamente en la cirugía de trasplantes pediátricos donde disminuir el tiempo de clampado es muy importante se necesitan métodos de sutura que aporten rapidez y facilidad de manejo al cirujano, es así que hemos planteado un nuevo estudio experimental donde frente a las técnicas de sutura convencionales continuas con polipropileno y Dexon, planteamos el uso de clips de titanio con el objetivo de conseguir su fácil y rápida aplicación en Autotrasplantes Renales Heterotópicos.

Material y métodos: Se utilizan 24 cerdos en crecimiento, que son sometidos a los 45 días de edad a un Autotrasplante Renal Heterotópico, con clips metálicos VCS, con técnicas de sutura convencionales continuas con polipropileno y con Dexon en anastomosis término-laterales de arteria y vena renal a la arteria aorta y vena cava.

Resultados: Confirmamos que los aplicadores de clips VCS son más fáciles de utilizar para el cirujano y disminuyen significativamente $(\mathrm{P} \leq 0,001)$ el tiempo empleado en las anastomosis término- laterales de las arterias renales a la arteria aorta

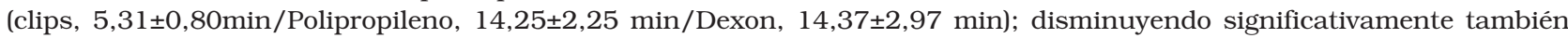
$(\mathrm{P} \leq 0,001)$ el tiempo empleado en las anastomosis término- laterales de las venas renales a la vena cava (clips, 8,25 $\pm 1,98$ min/Polipropileno, 16,25 $\pm 2,96 \mathrm{~min} /$ Dexon, 19,00 $\pm 4,50 \mathrm{~min}$ ).

Conclusiones: Los clips VCS aplicados en Autotrasplantes Renales Heterotópicos reducen significativamente con respecto a las suturas convencionales el tiempo empleado en realizar las reconstrucciones vasculares.

Palabras clave: Autotrasplante Renal Pediátrico. Clips metálicos VCS. Sutura. Tiempo de isquemia.

\section{Titanium metallic clips: easier and faster than conventional suturing for heterotopic renal} autotransplant in growing pigs
\end{abstract}

\begin{abstract} titanium clips in heterotopic renal autotransplants. artery and vein to the aorta and cava in an end-to-side fashion min/Polypropylene, 16.25 $\pm 2.96 \mathrm{~min} /$ Dexon, $19.00 \pm 4.50 \mathrm{~min}$ ). lar reconstruction, compared to conventional suturing.

Keywords: Paediatric Renal Autotransplant. VCS metallic clips. Suture. Ischemic time.
\end{abstract}

Introduction: When performing vascular reconstruction in growing elements, specifically in paediatric transplant surgery, where a short vascular occlusion time is mandatory, master and easily handled suturing methods are needed. Thus the present study compares conventional continuous suturing with polypropylene and dexon versus easier and faster to apply

Material and methods: 24 growing pigs were used for this study. Heterotopic renal autotransplant was performed when the animals were 45 days old using VCS clips, continous Polypropylene or Dexon suturing when anastomosing the renal

Results: VCS clips were easy to use for the surgeon, significantly $(\mathrm{P} \leq 0.001)$ decreasing the time needed for end-to-side anastomosis of the renal artery to the aorta (clips, $5.31 \pm 0.80 \mathrm{~min} /$ Polypropylene, $14.25 \pm 2.25 \mathrm{~min} /$ Dexon, $14.37 \pm 2.97$ $\mathrm{min}$ ); and also the time needed for end-to-side anastomosis of the renal veins to the cava (clips, $8.25 \pm 1.98$

Conclusions: The use of VCS clips in heterotopic renal autotransplants significantly decreases the time needed for vascu-

\begin{abstract}
A lgunos pacientes en edad pediátrica, bien por - desarrollar procesos patológicos, o por traumatismos, requieren procedimientos reconstructivos vasculares a consecuencia de las cuales surgen, en ocasiones, complicaciones tempranas o tardías; una de las más frecuentes es la falta de crecimiento en el lugar de la anastomosis vascular. Por ejemplo,
\end{abstract}

destacamos la coartación de la aorta, trasplantes y reimplantes en niños donde se requiere una reconstrucción quirúrgica en una etapa muy temprana de la vida. En este tipo de pacientes cobra importancia solucionar el problema con la falta de crecimiento en la zona de anastomosis y, con el exceso de tiempo de clampado y de intervención empleado. 
Los materiales que se utilizan para realizar suturas vasculares han experimentado una constante evolución. Concretamente en los vasos en crecimiento la cirugía vascular pediátrica sigue buscando alternativas a las complicaciones producidas con los distintos tipos de materiales y técnicas de sutura empleados en la actualidad. Una de las complicaciones más importante es la falta de crecimiento posterior a la anastomosis, que se produce en la técnica de sutura continua no reabsorbible, hecho que no ocurre con material reabsorbible, y sin embargo se corre el riesgo de otros problemas, también muy importantes, como la posibilidad de aneurismas a largo plazo y problemas con el tiempo de reabsorción.

En medio de esta preocupación y necesidad por mejorar los métodos de sutura de las anastomosis de los vasos en crecimiento, surge una línea de investigación basada en la aproximación discontinua de las superficies vasculares mediante clips metálicos de titanio no penetrantes.

Desde el año 2001, hasta la actualidad hemos publicado trabajos experimentales en vasos periféricos y aorta donde se estudia el comportamiento de los clips VCS. En estos trabajos demostramos experimentalmente que por su propiedad de no penetrar en la pared vascular los clips VCS no solo evitan el daño en la intima, la hiperplásia, favorecen la distensibilidad y el crecimiento del vaso ${ }^{1-6}$; sino que además aportan rapidez y facilidad de manejo al cirujano ${ }^{2,7-9}$; en contraposición a las técnicas de sutura convencionales.

Estos estudios nos aportan datos suficientes para seguir investigando el comportamiento de estos clips, que por las características y resultados descritos, podrían ser aplicados entre otros en trasplantes de órganos. Es por esta razón que una vez más nos hemos comprometido con un nuevo proyecto realizando Autotrasplantes Renales Heterotópicos en cerdos en plena etapa de desarrollo y crecimiento con clips VCS, con el fin de comprobar si la técnica de sutura con clips metálicos VCS se puede utilizar en trasplantes renales en pacientes pediátricos y si podría resultar ser un método más rápido de ejecutar para el cirujano que la técnica de sutura convencional continua con Polipropileno y continua con Dexon.

\section{MATERIAL Y MÉTODO}

El número de integrantes de este grupo experimental han sido 24 cerdos machos de 45 días de edad, sanos, de raza large-withe, la media de sus pesos fue de $9,77 \pm 1,80 \mathrm{Kg}$, todos ellos en plena fase de desarrollo y crecimiento.
Se utilizan los Aplicadores de Clips Vasculares denominados, Instrumentos VCS* que se componen de un cargador con varios clips independientes de titanio, sin vástagos, con capacidad automática de carga en las mandíbulas una vez disparada la anterior y de rotación del extremo.

Existen cuatro tamaños diferentes destinados a vasos de distintos diámetros pero nosotros en este trabajo hemos utilizado:

- El aplicador VCS M-0,9; tamaño del clip pequeño, distancia entre los extremos del clip antes de su cierre es de 0,9 mm, longitud total del clip es de 1,5 mm y en el cargador 40 clips.

- El aplicador VCS M-1,4; tamaño del clip mediano, distancia entre los extremos del clip antes de su cierre es de 1,4 mm, longitud total del clip es de 2,3 $\mathrm{mm}$ y en el cargador 35 clips.

Además disponemos de elementos accesorios como son la pinza de aproximación de los bordes del vaso y la pinza de extracción para extraer los clips mal colocados.

Las técnicas quirúrgicas se han llevado a término en los quirófanos habilitados para tal fin en el Centro de Cirugía de Mínima Invasión “Jesús Usón”. El alojamiento, cuidados, mantenimiento y seguimiento preoperatorio y postoperatorio de los animales de esta experiencia, se han efectuado en el animalario de este Centro, el cual está acreditado como Centro usuario de animales de experimentación (CC-001), por la Consejería de Agricultura y Medio Ambiente de la Junta de Extremadura. A lo largo del estudio se siguieron las normativas europeas y españolas referentes a animales vertebrados de experimentación. Siendo este ensayo experimental aprobado por el Comité Ético de Bienestar Animal del Centro de Cirugía de Mínima Invasión “Jesús Usón”.

\section{Método}

Los 24 cerdos, son sometidos a un Autotrasplante Renal Heterotópico, sin transposición del uréter, realizando transposiciones del riñón derecho desde su localización habitual a la zona craneal a la fosa ilíaca, efectuando 8 anastomosis vasculares termino-laterales con clips metálicos VCS de las arterias renales y 8 de venas renales con la arteria aorta y vena cava respectivamente (Fig. 1A), 8 con técnicas de sutura continua con Polipropileno (Fig. 1B) y 8 con Dexon (Fig. 1C). Posteriormente todos los animales permanecerán en crecimiento durante 6 meses. 


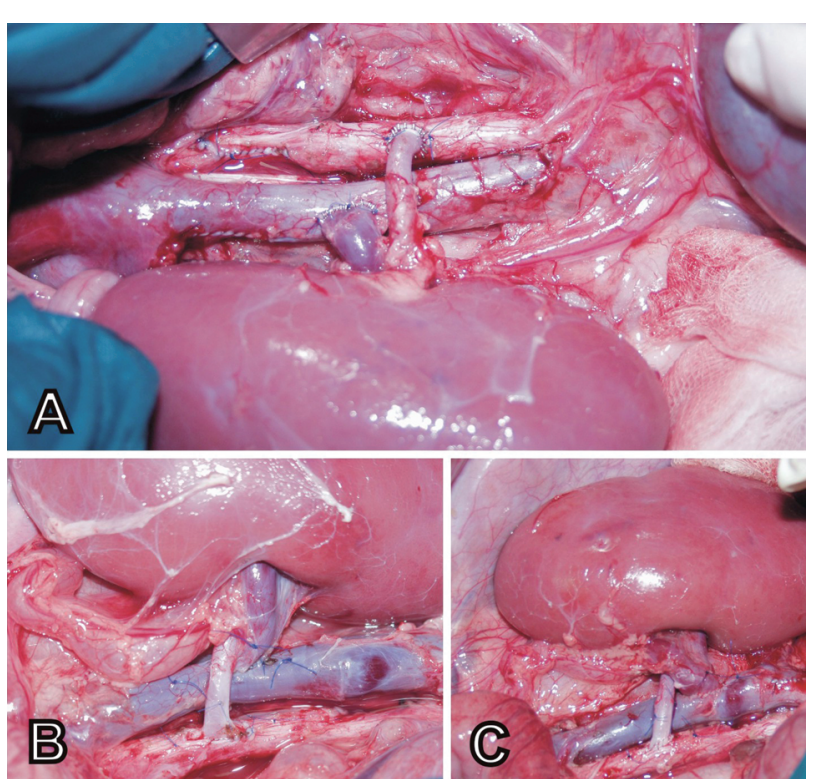

FIGURA 1. Autotrasplante Renal Heterotópico con Clips (A), con sutura continua con Polipropileno (B) y con Sutura continua con Dexon (C).

La técnica de anastomosis vascular con clips metálicos VCS la comenzamos con la colocación de dos puntos guía en las arterias y tres en las venas que nos sirven de aproximación de los dos extremos vasculares y además disminuyen la tensión. Posteriormente colocamos los clips en la cara anterior, volteamos el vaso $180^{\circ} \mathrm{y}$ finalmente completamos la anastomosis con la aplicación de los clips en la cara posterior. Todas las anastomosis han sido realizadas por el mismo cirujano abalado por una amplia experiencia en microcirugía vascular, con un entrenamiento y una curva de aprendizaje previa no solo en la aplicación de los clips VCS, con los que ha realizado varios trabajos experimentales de los cuales algunos han sido publicados con anterioridad (18), sino también en suturas vasculares.

Confirmamos mediante técnicas de medición intraoperatorias el tiempo empleado en cada reconstrucción término-lateral vascular, arterial y venosa, esto englobaría el tiempo empleado en la colocación de los puntos guía (en la técnica con clips), anastomosis de la cara anterior del vaso y anastomosis de la cara posterior, teniendo en cuenta (en los casos que ha sido necesario) el tiempo empleado en la reparación de la permeabilidad de la anastomosis, con la colocación de clips o suturas adicionales, midiendo también el tiempo de clampado total de las arterias y venas.

Consideramos que las diferencias en el tiempo empleado en toda la intervención con una $u$ otra técnica vienen marcadas por las diferencias de tiempo empleado en las reconstrucciones vasculares con las diferentes técnicas de sutura empleadas.

\section{Mecánica e incidencias intraoperatorias}

No existen diferencias estadísticamente significativas entre los diámetros de las arterias y venas intervenidas con clips y las intervenidas con sutura preintervención. La media del diámetro interno de las arte-

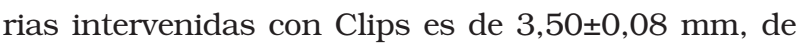
las arterias intervenidas con sutura (Polipropileno) es $2,80 \pm 0,55 \mathrm{~mm}$ y de las arterias intervenidas con sutura (Dexon) de 3,72 $\pm 1,28 \mathrm{~mm}$. La media del diámetro interno de las venas intervenidas con clips es de $5,67 \pm 1,16 \mathrm{~mm}$, de las venas intervenidas con sutura (polipropileno) es 6,00 $\pm 0,70 \mathrm{~mm}$ y de las venas

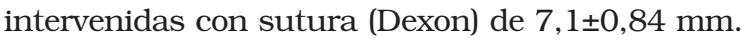

Hemos colocado en las arterias 16-18 clips $(17,62 \pm 1,96$ clips de media) en los 8 animales intervenidos, y 19-21 clips $(20,50 \pm 2,05$ clips de media) en las venas, así como técnica de sutura continua tanto con sutura con polipropileno como con Dexon. Todos los clips colocados en la arteria para realizar la anastomosis fueron de tamaño mediano (aplicador VCS M-1,4 mm) y en la vena de tamaño pequeño (aplicador VCS M-0,9 mm).

\section{Estudio estadístico}

Cada una de las variables previamente indicadas se describen mediante la media desviación estándar.

En el factor tiempo, en caso de existir diferencias estadísticamente significativas entre los distintos grupos, las diferencias entre medias se establecieron a posteriori mediante una prueba de Tukey a un nivel de significación $\mathrm{p}<0,05$.

Por otro lado, la posible existencia de diferencias estadísticamente significativas entre los tiempos quirúrgicos medidos, debidas al tipo de vaso intervenido (vena y arteria) y debidas al tipo de técnica de sutura empleada (técnica de sutura con Clips y técnica de sutura con Polipropileno y Dexon) se determinó mediante un análisis de varianza bifactorial.

\section{RESULTADOS}

1. Tiempos empleados en realizar las anastomosis termino-laterales de las arterias y venas con Clips, Dexon y Polipropileno.

El tiempo medio que se emplea en la reconstrucción de la anastomosis termino-lateral de la arteria renal a la arteria aorta y de la vena renal a la vena 
cava con Clips es significativamente menor al tiempo empleado en la reconstrucción con técnica de sutura continua con Dexon y con Polipropileno, no existiendo diferencias significativas entre estas dos últimas técnicas (Tablas 1 y 2) (Figs. 2 y 3). Los tiempos de las diferentes etapas en las que hemos dividido la reconstrucción realizada tanto en las arterias como en las venas, mostraron también diferencias estadísticamente significativas debidas al tipo de técnica de sutura empleada (clips y sutura) (Tablas 1 y 2) (Figs. 2 y 3). El tiempo empleado en la colocación de los puntos guía necesarios exclusivamente para la realización de la técnica con Clips, es de $2,12 \pm 0,44 \mathrm{~min}$ en arterias y de $3,00 \pm 0,75 \mathrm{~min}$ en venas.

2. Comparación entre los tiempos empleados en realizar las anastomosis termino-laterales entre las arterias y las venas.

Los tiempos de las diferentes eta-

Tabla 1. Tiempo empleado en la reconstrucción vascular y tiempo de clampado en pas en las que hemos dividido la anastomosis de arterias renales y aorta realizadas con Clips VCS, sutura continua reconstrucción realizada en la con Dexon y continua con Polipropileno

\begin{tabular}{llccc}
\hline $\begin{array}{l}\text { Pasos de } \\
\text { Anastomosis }\end{array}$ & $\begin{array}{l}\text { La técnica de } \\
\text { Sutura }(*)\end{array}$ & $\begin{array}{c}\text { Tiempos } \\
\text { (min) }\end{array}$ & Valor de p & Sutura \\
\hline Cara anterior & Polipropileno & $5,37 \pm 0,91$ & $\mathrm{p}<0,001$ & $\mathrm{a}$ \\
& Ac. Poliglicólico & $5,00 \pm 1,07$ & $\mathrm{p}<0,001$ & $\mathrm{a}$ \\
& Clips & $0,87 \pm 0,15$ & $\mathrm{p}<0,001$ & $\mathrm{~b}$ \\
Cara posterior & Polipropileno & $7,00 \pm 1,41$ & $\mathrm{p}<0,001$ & $\mathrm{a}$ \\
& Ac. Poliglicólico & $7,62 \pm 1,76$ & $\mathrm{p}<0,001$ & $\mathrm{a}$ \\
& Clips & $1,38 \pm 0,38$ & $\mathrm{p}<0,001$ & $\mathrm{~b}$ \\
Total anastomosis & & & & \\
& Polipropileno & $14,25 \pm 2,25$ & $\mathrm{p}<0,001$ & $\mathrm{a}$ \\
& Ac. Poliglicólico & $14,37 \pm 2,97$ & $\mathrm{p}<0,001$ & $\mathrm{a}$ \\
Tiempo clampado & Clips & $5,31 \pm 0,80$ & $\mathrm{p}<0,001$ & $\mathrm{~b}$ \\
& & & & \\
& Polipropileno & $16,37 \pm 2,97$ & $\mathrm{p}<0,001$ & $\mathrm{a}$ \\
& Ac. Poliglicólico & $16,25 \pm 2,25$ & $\mathrm{p}<0,001$ & $\mathrm{a}$ \\
& Clips & $7,31 \pm 0,80$ & $\mathrm{p}<0,001$ & $\mathrm{~b}$ \\
\hline
\end{tabular}

$(*)$ Efecto significativo para el tipo de técnica de sutura $(\mathrm{p}<0,001)$.

Test de Tukey, a,b,.- letras distintas denotan medias estadísticamente diferentes.

Todos los datos están expresados como media \pm desviación estándar.

Tabla 2. Tiempo empleado en la reconstrucción vascular y tiempo de clampado en anastomosis de venas renales y cava realizadas con Clips VCS, sutura continua con Dexon y continua con Polipropileno

\begin{tabular}{llccc}
\hline $\begin{array}{l}\text { Pasos de } \\
\text { Anastomosis }\end{array}$ & $\begin{array}{l}\text { La técnica de } \\
\text { Sutura (*) }\end{array}$ & $\begin{array}{c}\text { Tiempos } \\
\text { (min) }\end{array}$ & Valor de p & Sutura \\
\hline Cara anterior & Polipropileno & $6,00 \pm 1,41$ & $\mathrm{p}<0,05$ & $\mathrm{a}$ \\
& Ac. Poliglicólico & $5,87 \pm 2,23$ & $\mathrm{p}<0,05$ & $\mathrm{a}$ \\
& Clips & $2,00 \pm 0,93$ & $\mathrm{p}<0,05$ & $\mathrm{~b}$ \\
Cara posterior & Polipropileno & $8,5 \pm 1,42$ & $\mathrm{p}<0,001$ & $\mathrm{a}$ \\
& Ac. Poliglicólico & $10,75 \pm 2,18$ & $\mathrm{p}<0,001$ & $\mathrm{a}$ \\
& Clips & $2,25 \pm 1,00$ & $\mathrm{p}<0,001$ & $\mathrm{~b}$ \\
Total anastomosis & Polipropileno & $16,25 \pm 2,96$ & $\mathrm{p}<0,001$ & $\mathrm{a}$ \\
& Ac. Poliglicólico & $19,00 \pm 4,50$ & $\mathrm{p}<0,001$ & $\mathrm{a}$ \\
& Clips & $8,25 \pm 1,98$ & $\mathrm{p}<0,001$ & $\mathrm{~b}$ \\
& & & & $\mathrm{a}<0,001$ \\
Tiempo clampado & Polipropileno & $18,37 \pm 2,63$ & $\mathrm{p}<0,001$ & $\mathrm{a}$ \\
& Ac. Poliglicólico & $21,03 \pm 2,89$ & $\mathrm{p}<0,001$ & $\mathrm{~b}$ \\
\hline
\end{tabular}

(*) Efecto significativo para el tipo de técnica de sutura $(\mathrm{p}<0,001)$.

Test de Tukey, a,b,.- letras distintas denotan medias estadísticamente diferentes.

Todos los datos están expresados como media \pm desviación estándar. arteria y en la vena, y el tiempo total como hemos descrito anteriormente mostraron diferencias estadísticamente significativas debidas al tipo de técnica de sutura empleada (clips y sutura); exceptuando el tiempo empleado en la colocación de los puntos guía en el que no hubo diferencias estadísticamente significativas debidas a que solamente se utiliza este paso en la técnica con clips, ni tampoco diferencias estadísticamente significativas debido al tipo de vaso.

Así, confirmamos que el tiempo empleado en la reconstrucción de los vasos tanto con clips como con las suturas es mayor en la vena que en la arteria pero con diferencias estadísticamente significativas solamente en el grupo con Dexon $(\mathrm{p}<0,05)$.

En el resto de los pasos de la reconstrucción vascular (sutura de la cara anterior y la cara posterior) el tiempo empleado es mayor en la vena que en la arteria, tanto con la utilización de los clips como con el resto de suturas, pero solamente con diferencias estadísticamente significativas debidas al tipo de vaso $(p<0,05)$ en la colocación de la cara posterior en el grupo Dexon.

3. Tiempos de Clampado de arterias y venas en el autotrasplante.

El tiempo medio de hemostasia o 


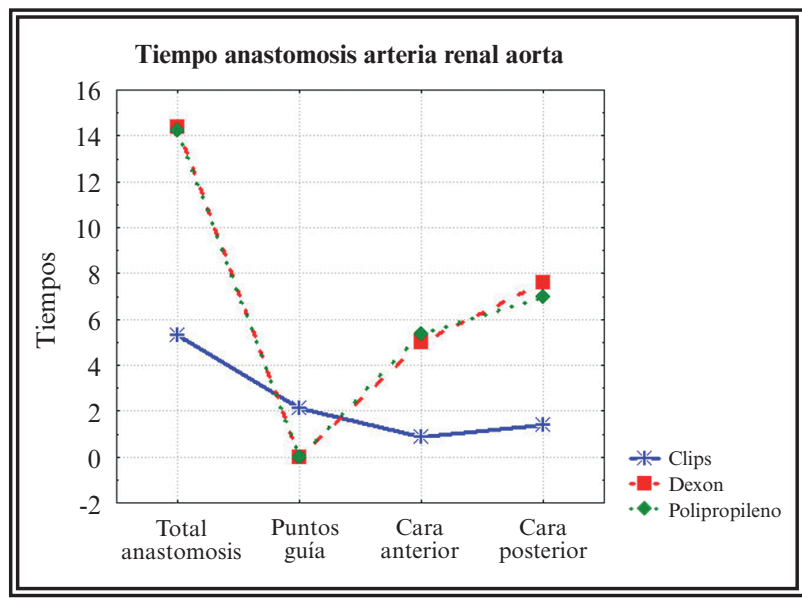

FIGURA 2. Tiempo quirúrgico empleado en los distintos pasos de la reconstrucción de las arterias con Clips, sutura continua con Dexon y sutura continua con Polipropileno.

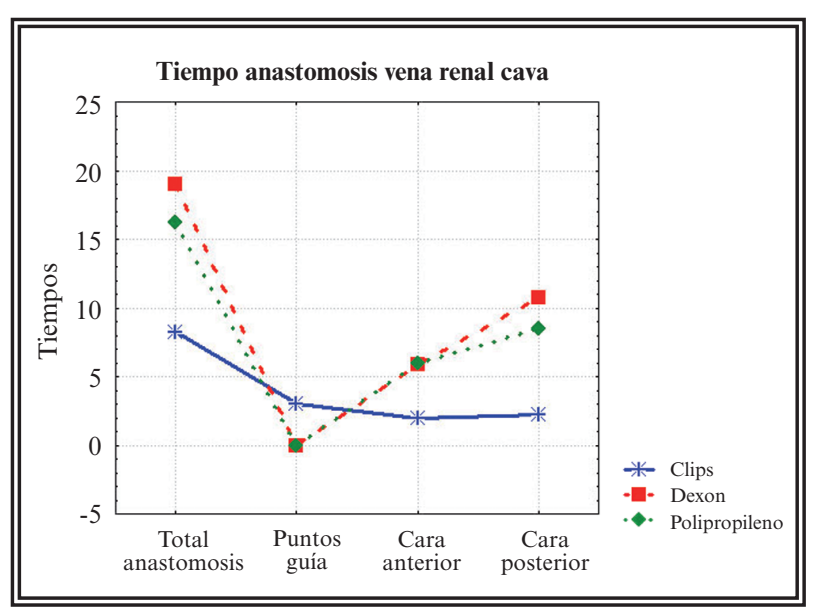

FIGURA 3. Tiempo quirúrgico empleado en los distintos pasos de la reconstrucción de las venas con Clips, sutura continua con Dexon y sutura continua con Polipropileno.

clampado utilizado para la reconstrucción total de la anastomosis arteria renal-aorta con Clips es de $07,31 \pm 0,80 \mathrm{~min} y$ es significativamente menor $(\mathrm{p}<0,001)$ al tiempo empleado con sutura continua con Dexon (16,25 $\pm 0,80 \mathrm{~min})$ y con sutura continua con Polipropileno $(16,37 \pm 2,97 \mathrm{~min})$, no existiendo diferencias estadísticamente significativas entre el tiempo de clampado con sutura continua con Dexon y con Polipropileno (Tablas 1 y 2) (Fig. 4).

Por otro lado el tiempo medio de hemostasia o clampado utilizado para la reconstrucción total de la anastomosis vena renal-cava con Clips es de $09,84 \pm 1,58 \mathrm{~min} y$ es significativamente menor $(\mathrm{p}<0,001)$ al tiempo empleado con sutura continua con Dexon $(21,03 \pm 2,89 \mathrm{~min})$ y con sutura continua con polipropileno $(18,37 \pm 2,63 \mathrm{~min})$, no existiendo

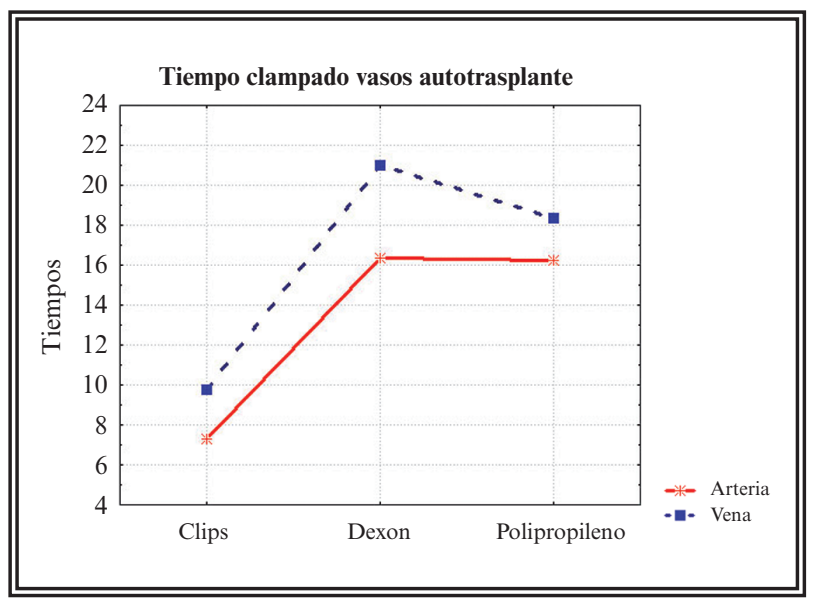

FIGURA 4. Tiempo de clampado empleado en la reconstrucción de las arterias y venas con Clips, sutura continua con Dexon y sutura continua con Polipropileno.

diferencias estadísticamente significativas entre el tiempo de clampado con sutura continua con Dexon y con Polipropileno.

Confirmamos además que el tiempo de clampado tanto con clips como con las suturas es significativamente mayor en la vena que en la arteria en los grupos Clips y Dexon $(\mathrm{p}<0,05)$, pero sin diferencias estadísticamente significativas debidas al tipo de vaso en el grupo Polipropileno.

\section{DISCUSIÓN}

Los resultados dependientes del tipo de vaso son debidos al mayor diámetro de la vena y en consecuencia a la necesidad de colocar un mayor número de clips y suturas en esta, siendo también un factor importante la mayor dificultad de la técnica.

Por otro lado existen diferencias de tiempo con el empleo de las diferentes técnicas de sutura en la cara posterior de los vasos debido a la mayor dificultad que conlleva la realización de esta cara. En la técnica convencional esta cara se sutura por la cara luminal del vaso, para no movilizar el riñón, técnica quirúrgica con mas más dificultad sobre todo con Dexon que conlleva, por su característica innata de producir fricción al deslizarse por el vaso, un mayor cuidado y delicadeza en su colocación. Por otro lado en la técnica con clips tenemos inevitablemente que movilizar el riñón para la colocación de los clips resultando más dificultosa la técnica quirúrgica en esta cara por la tensión del vaso y la posición forzada del cirujano.

Muy importante en este trabajo son los tiempos empleados con los clips; estamos mejorando el tiem- 
po de nuestros primeros trabajos ${ }^{2,7,8}$ en anastomosis término-terminal, técnica menos laboriosa que la témino-lateral de este trabajo. Hemos reducido el tiempo tres veces menos en las reconstrucciones de anastomosis arteriales y a la mitad en las venosas. Con este hecho seguimos demostramos al igual que varios autores como Zeebregts et $\mathrm{al}^{10}$ que la técnica con clips VCS, como toda técnica quirúrgica, está sometida a un aprendizaje previo y a un entrenamiento y que además la curva de aprendizaje no en muy larga como aseguran Leppäniemi et $\mathrm{al}^{11}$ y Nataf et $\mathrm{al}^{12}$.

Son ya muchos los autores que describen en sus trabajos, una disminución, estadísticamente significativa o no, en el tiempo empleado en las recons-

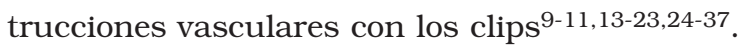

Resaltamos también dos trabajos donde se demuestran diferencias en el tiempo de clampado y de isquemia empleando los clips y la sutura, se trata del trabajo de Zeebregts et al. ${ }^{37}$ realizado en arterias carótidas y aorta, y el trabajo de Jones ${ }^{38}$ en trasplantes renales, este último describe una disminución importante en el tiempo de isquemia empleado en la realización de trasplantes renales con anastomosis de la arteria y vena renales a los vasos ilíacos con la utilización de los clips con respecto a la sutura.

Destacamos una semejanza con nuestro estudio de dos trabajos ${ }^{24,35}$ donde los autores también describen una reducción importante del tiempo empleado con los clips en comparación con sutura continua.

Sin embargo existen dos trabajos en la bibliografía consultada donde los autores describen similitud en el tiempo empleado con clips y suturas, se trata de los trabajos de Dal Ponte et al. ${ }^{39}$ en fístulas arteriovenosas en la arteria y venas femorales con prótesis de politetrafluoroetileno (PTFE) con sutura continua con polipropileno y clips, y de otro trabajo realizado por Geevarchese et al. ${ }^{40}$ en anastomosis de arteria hepática y de la vena porta en trasplante hepático con clips VCS y con sutura continua.

\section{CONCLUSIONES}

En consecuencia los resultados de este trabajo podrían poner de manifiesto una importante mejora para la cirugía vascular pediátrica al aplicar un método de sutura que pone al alcance del cirujano una técnica fácil y rápida de realizar, disminuyendo en consecuencia los tiempos de clampado en el campo del trasplante y del reimplante infantil.

\section{Agradecimientos}

Nuestro especial agradecimiento a la Fundación Mutua Madrileña por la financiación de este proyecto.

A todo el equipo anestésico encabezado por Juan Rafael Lima y al equipo de Técnicos de Quirófano del Centro de Cirugía de Mínima Invasión "Jesús Usón" que han contribuido a que este trabajo se lleve a cabo.

\section{REFERENCIAS}

1. Carmen Calles M, Lima MA, Viguera FJ et al. Clips metálicos VCS, una novedosa técnica de sutura que permite el crecimiento longitudinal y transversal de los vasos: avance preliminar. Patologia Vascular Julio-Septiembre 2001;7(3):535-542.

2. Carmen Calles M, Lima MA, Crisóstomo V, Usón JM, Usón J. End-to-end anastomosis in growing vessels using a novel suturing technique: VCS metallic staples. Ann Vasc Surg. 2002;16 (3):345-352.

3. Lima MA, Calles C, Lima JR, Martin MF, Crisostomo V, Hernandez FJ, et al. Ultrasonographic experimental study on the evolution of vascular anastomosis with metallic clips in growing sheep carotid artery. Vet Radiol Ultrasound. 2002;43(2): 171-177.

4. Calles-Vázquez MC, Usón JM, Viguera FJ, Sun F, Paz JI, UsónGargallo J. VCS clips versus polypropylene suture in end-toend anastomosis of growing arteries and veins. Annals of Vascular Surgery. 2005;19(3):320-327.

5. Calles-Vázquez Mdel C, Viguera FJ, Sun F, Usón JM, Usón J. Vein and artery growth following anastomosis with "ves" clips versus interrupted polypropylene suture: application in pediatric vascular surgery. Journal of Pediatric Surgery. 2005;40(9); 1428-1435.

6. Calles-Vázquez MC, Crisóstomo V, Sun F, Usón-Gargallo J. Angiographic, ultrasonographic, and macroscopic assessment of aortic growth after VCS clips, interrupted polypropylene, or running polyglycolic acid anastomosis. J Pediatr Surg. 2007;42(10): 1695-1702.

7. Calles Vázquez MC, Usón Casaús $\mathrm{JM}^{\mathrm{a}}$, Lima Rodríguez MA, et al. Clips metálicos VCS: una técnica de sutura más rápida y fácil de utilizar que la sutura convencional en reconstrucciones de vasos en crecimiento. Angiologia. 2003;55(6):497-509.

8. Calles-Vázquez MC, Viguera FJ, Crisóstomo V, Usón-Gargallo J. Vascular Closure Stapler Clip Anastomosis Decreases aortic Cross-Clamping Time Compared to Interrupted Nonabsorbable and Running Absorbable Sutures in Growung Pigs. Annals of Vascular Surgery. 2006;20(1):35-41.

9. Pikoulis E, Burris D, Rhee P, Nishibe T, Leppäniemi A, Wherry D, et al. Rapid Arterial Anastomosis with Titanium Clips. Am J Surg. 1998;175(6):494-496.

10. Zeebregts CJ, van den Dungen JJ, Kalicharan D, Cromheecke M, van der Want J, van Schilfgaarde R. Nonpenetrating vascular clips for small-caliber anastomosis. Microsurgery. 2000;20(3):131-138.

11. Leppäniemi A, Rich N, Pikoulis E, Rhee P, Burris D, Wherry D. Sutureless vascular reconstruction with titanium clips. Int Angiol. 2000;19(1):69-74

12. Nataf P, Kirsch W, Hill AC, Anton T, Zhu YH, Ramadan R, et al. Nonpenetrating clips for coronary anastomosis. Ann Thorac Surg. 1997;63(6 Suppl):S135-137.

13. Ahn SS, Clem MF, Braithwaite BD et al. Laparoscopic aortofemoral bypass. Initial experence in an animal model. Ann Surg. 1995;222(5):577-683.

14. Akita M, Takenaka H, Harada M, Li TS, Zhang H, Zempo N, et al. Can nonpenetrating vascular closure staples and hepatocyte growth factor prevent intimal hyperplasia following ePTFE grafting of the carotid artery in rabbits?. Surg Today. 2002; 32(7):618-622. 
15. Boeckx WD, Darius O, van den hof B, van Holder C. Scanning electron microscopic analysis of the stapled microvascular anastomosis in the rabbit. Ann Thorac Surg. 1997;63(6 Suppl): S128-134.

16. Cope C, Lee K, Stern H, Pennington D. Use of the VCS clip applier for microvascular anastomosis in free flap surgery. Plast Reconstr Surg. 2000;106(1):107-110.

17. Dimakakos PB, Pafiti-Kondi A, Doufas A et al. Venous repair with vascular clips and conventional suture: A comparative experimental study. Phlebology 1999; 14: 65-70.

18. Findlay JM, Megyesi JF. Carotid arteriotomy closure Using a vascular clip system. Neurosurgery. 1998;42(3):550-554.

19. Kirsch WM, Zhu YH, Hardesty RA, Chapolini R.. A new Method for Microvascular anastomosis: Report of Experimental and clinical Research. Am Sugr. 1992;58(12):722-727.

20. Kirsch WM, Zhu YH, Boukouvalas $Z$ et al. Morphologic Events During Healing of Microvascular anastomosis. En Sun Lee, ed. Color atlas of Microsurgery. Missouri (Japan): Ed. Ishiyaku EuroAmerica. 1993. p. 79-88.

21. Kirsch WM, Zhu YH, Gaskill D, Stewart S, Hardesty RA, Lyons TL. Tissue reconstruction with Nonpenetrating Arcuate-Legged Clips. (Potencial Endoscopic Aplications). J Reprod Med. 1992; 37(7):581-586.

22. Leppäniemi A, Wherry D, Pikoulis E, Hufnagel H, Waasdorp C, Fishback N, et al. Arterial and venous repair with vascular clips: comparison with suture closure. J Vasc Surg. 1997;26(1): 24-28.

23. Rampillon F, Hailaud YM, Malka G, Justrabo E.. Sutures Microchirurgicales par agrafes non transfixiantes. Étude experimentale sur quinze aortes de rat. Ann Chir Plast Esthet. 1996;41(4):376-380.

23. Rampillon F, Hailaud YM, Malka G, Justrabo E.. Sutures Microsurgical sutures with non-transfixing staples. An experimental study of 15 rat aortas. Ann Chir Plast Esthet. 1996;41(4):376-380.

24. Baguneid MS, Goldner S, Fulford PE, Hamilton G, Walker MG, Seifalian AM. A comparison of para-anastomotic compliance profiles after vascular anastomosis: Nonpenetrating clips versus standard sutures. J vasc Surg. 2001;33(4):812-820.

25. Caiati JM, Madigan JD, Bhagat G, Benvenisty AI, Nowygrod R, Todd GJ.. Vascular clips have no significant effect on the cellular proliferation, intimal changes, or peak systolic velocity at anastomoses in rabbit vein grafts. J Surg Res. 2000;92(1):29-35.

26. Deb S, Martin B, Sun L, Burris D, Wherry D, Pikoulis E, et al. Comparison of titanium vascular closure staples with suture repair of the thoracic aorta in swine. Chest. 2000;118(6):17621768.

27. Ducasse E, Basseau F, Puppinck P, Baquey C. Can minimal arterial aggressions using non-penetrating mechanical clip suture prevent myo-intimal hyperplasia?. Preliminary results. J Mal Vasc. 2001;26(1):50-54.

28. Gerbault O, Arrouvel C, Servant JM, Revol M, Banzet P. VCS microclip anastomosis on blood vessels of less than 2 millimeters in diameter. Preliminary experimental study in the rat. Ann Chir Plast Esthet. 1998;43(1):27-39.
29. Golling M, Mehrabi A, Weiss G, Schäffer F, Kraus T, Klar E. The VCS clip-experimental experiences with a new vascular suture stapling device. Chirurg. 1999;70(2):206-210.

30. Lambert F, Couturaud B, Cruel T, Lecoin G, Cariou JL. Vascular microanastomosis by eversion and stapling using VCS forceps. Presentation of the technique and experimental evaluation of its reliability. Ann Chir Plast Esthet. 1998;43(1): 14-26.

31. Lee JW, Choo SJ, Oh JH, Lee I, Kwon YM, Lee YJ, et al. Anastomosis of vessels less than $2 \mathrm{~mm}$ with the vascular clip system clip applier. J Korean Med Sci. 2001;16(3):303-308.

32. Payne CE, Hunt SP, Lamberty BG. Primary sciatic nerve repair using titanium staples. Br J Plast Surg. 2002;55(4):330-334.

33. Pikoulis E, Koronarchis D, Filis K, Leppäniemi A, Papas S, Xiromeritis N, et al. Study comparing sutures and nonpenetrating titanium clips for arteriotomy closure after embolectomy. Surg Endosc. 2001;15(7):726-728.

34. Pikoulis E, Rhee P, Nishibe T, Leppäniemi AK, Fishback N, Hufnagel HV, et al. Arterial reconstruction with vascular clips is safe and quicker than sutured repair. Cardiovasc Surg. 1998;6(6):573-578.

35. Pikoulis E, Rhee $\mathrm{P}$, Nishibe T, Burris D, Leppäniemi AK, Fishback N, et al. Arterial repair with synthetic patch by using titanium clips. J Trauma. 2000;48(2):292-295.

36. Zhu YH, Kirsch WM, Cushman R et al. Comparison of suture and clip for microvascular anastomoses. Surg Forum. 1985; 36:492-495.

37. Zeebregts C, van den Dungen J, Buikema H, van der Want J, van Schilfgaarde R. Preservation of endothelial integrity and function in experimental vascular anastomosis with non-penetrating clips. Br J Surg. 2001;88(9):1201-1208.

38. Jones JW. A new anastomotic technique in renal transplants reduces warm ischemia time. Clin Transplant 1998;12(1):7072.

39. Dal Ponte DB, Berman SS, Patula VB et al. Anastomotic tissue response associated with expanded polytetrafluoroethylene access gratts constructed by using nonpenetrating clips. J Vasc Surg. 1999; 30:325-333.

40. Geevarghese SK, Bradley AL, Atkinson J, Wright JK, Chapman WC, Van Buren DH, et al. Comparison of arcuate-legged clipped versus sutured hepatic artery, portal vein, and bile duct anastomoses. Am Surg. 1999;65(4):311-316.

Correspondencia autor: Dra. $\mathrm{M}^{\mathrm{a}}$ Carmen Calles Vázquez Unidad de Microcirugía.

Centro de Cirugía de Mínima Invasión "Jesús Usón”

Ctra. N-521, Km. 41,8. 10071 - Cáceres.

Tel.: 927181032

E-mail autor: mccalles@ccmijesususon.com

Información artículo: Experimental trasplante

Trabajo recibido: septiembre 2008

Trabajo aceptado: octubre 2008 